archives-ouvertes

\title{
Is the word-length effect linked to subvocal rehearsal?
}

\author{
Charlotte Jacquemot, Emmanuel Dupoux, Anne-Catherine Bachoud-Lévi
}

\section{To cite this version:}

Charlotte Jacquemot, Emmanuel Dupoux, Anne-Catherine Bachoud-Lévi. Is the wordlength effect linked to subvocal rehearsal?. Cortex, Elsevier, 2011, 47 (4), pp.484-493. 10.1016/j.cortex.2010.07.007 . hal-02326598

\section{HAL Id: hal-02326598 \\ https://hal.archives-ouvertes.fr/hal-02326598}

Submitted on 19 Jan 2021

HAL is a multi-disciplinary open access archive for the deposit and dissemination of scientific research documents, whether they are published or not. The documents may come from teaching and research institutions in France or abroad, or from public or private research centers.
L'archive ouverte pluridisciplinaire HAL, est destinée au dépôt et à la diffusion de documents scientifiques de niveau recherche, publiés ou non, émanant des établissements d'enseignement et de recherche français ou étrangers, des laboratoires publics ou privés. 


\section{A R T I C L E I N F O}

Article history:

Received 30 July 2009

Reviewed 15 December 2009

Revised 4 May 2010

Accepted 14 July 2010

Action editor Roberto Cubelli

Published online $\mathrm{xxx}$

Keywords:

Phonological short-term memory

Subvocal rehearsal

Word-length effect

Articulatory suppression

Conduction aphasia

\begin{abstract}
A B S T R A C T
Models of phonological short-term memory (pSTM) generally distinguish between two components: a phonological buffer and a subvocal rehearsal. Evidence for these two components comes, respectively, from the phonological similarity effect and the wordlength effect which disappears under articulatory suppression. But alternative theories posit that subvocal rehearsal is only an optional component of the pSTM. According to them, the depletion of the length effect under articulatory suppression results from the interference of the self-produced speech rather than the disruption of subvocal rehearsal.

In order to disentangle these two theories, we tested two patients with a short-term memory deficit. FA, who presents a pseudoword repetition deficit, and FL, who does not. FA's deficit allowed for the observance of an ecological case of subvocal rehearsal disruption without any articulatory suppression task. FA's performance in pSTM tasks reveals as controls a phonological similarity effect, and contrary to controls no word-length effect. In contrast, the second patient, FL, exhibits the same effects as control subjects. This result is in accordance with models of pSTM in which the word-length effect emerges from subvocal rehearsal and disappears when this latter is disrupted.
\end{abstract}

\section{Introduction}

Phonological short-term memory (pSTM) allows the temporary storage and processing of verbal information. The first models of pSTM were developed in the 1970s (Baddeley and Hitch, 1974) but some components of this system are still discussed. Models of pSTM generally distinguish between two components: a phonological buffer and a subvocal rehearsal process (Baddeley, 1986). The phonological buffer is assumed to transiently store the phonological input and subvocal rehearsal to refresh the memory traces stored in the phonological buffer before they decay. Thus subvocal rehearsal increases the storage capacity of PSTM beyond the capacity of the phonological buffer itself (Cowan, 2001). Recent findings support a revision of the standard model, which describes a single phonological buffer, to a new model with two separate phonological buffers: an input buffer (in the perception system) and an output buffer (in the production system) that store phonological input and output respectively (Nickels et al., 1997; Martin et al., 1999; Laganaro and Alario, 2006). Subvocal rehearsal arises from the circulation of phonological information between these two buffers and

\footnotetext{
* Corresponding author. Laboratoire de Sciences Cognitives et Psycholinguistique, Ecole Normale Supérieure, 29, rue d'Ulm, 75005 Paris, France.

E-mail address: charlotte.jacquemot@ens.fr (C. Jacquemot). 0010-9452/\$ - see front matter doi:10.1016/j.cortex.2010.07.007
} 


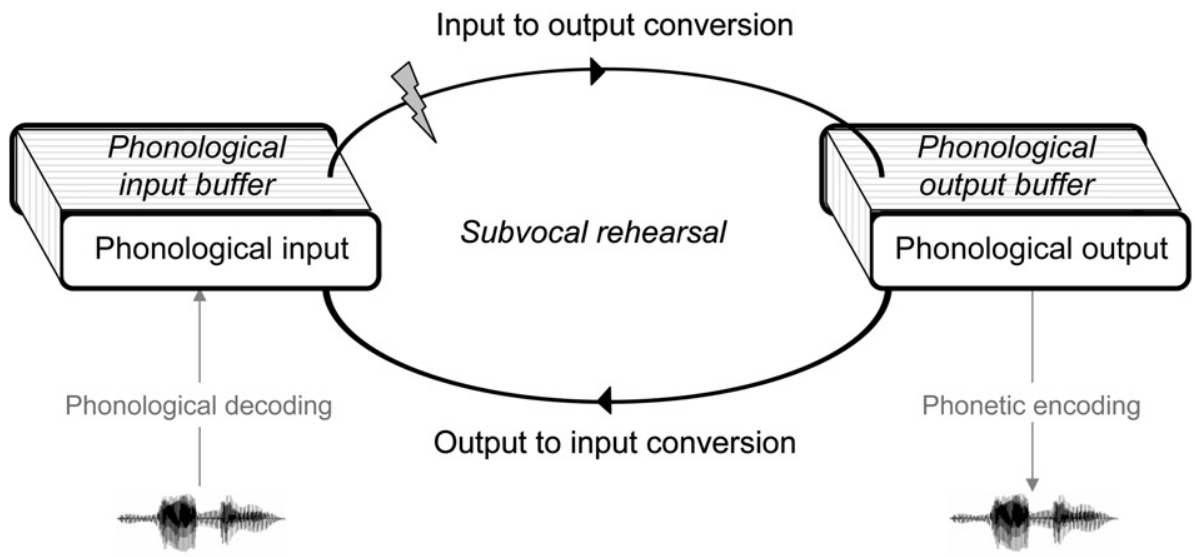

Fig. 1 - Functional model of phonological STM based on Jacquemot and Scott (2006). After the initial acoustic analysis, the phonological decoding is defined as the translation of acoustic information into discrete segmental categories that belong to the language, i.e., the phonological input and that can be stored in the phonological input buffer. The phonological encoding transforms the phonological output stored in the phonological output buffer into a motor programme for producing aloud the word. In this model, pSTM is composed of the two buffers dedicated to phonological processing in perception and production (phonological input and output buffers), and the mechanisms that convert phonological input information into output and vice versa (subvocal rehearsal). The locus of FA's deficit is modelled on the conversion mechanism of phonological input into phonological output.

involves two conversion mechanisms, one that converts phonological input into phonological output and one that does the reverse mapping (see Fig. 1) (Jacquemot and Scott, 2006). Previous studies suggest that these two buffers are not functionally equivalent and may be differentially affected by phonological variables like phonological similarity and phonological length.

The phonological input buffer has been found to be highly sensitive to phonological similarity: lists of phonologically similar stimuli are less well recalled than lists of dissimilar ones (Baddeley et al., 1984; Vallar and Baddeley, 1984a). For instance a list containing / bim/, / pim/, / kim/ yields more errors than a list containing / $\mathrm{bam} /$, /liv/, /ron/. This effect is called the phonological similarity effect and is present even when subvocal rehearsal is blocked by articulatory suppression (Baddeley, 1986). Typically, articulatory suppression is done by asking participants to continuously repeat a token (e.g., "the, the, the") during the memory task.

In contrast, the phonological output buffer is known to be highly sensitive to phonological length (Shallice et al., 2000; Q1 Nickels and Howard, 2004; Caramazza et al., 1986). In a very detailed analysis of aphasic speech production errors, Nickels and Howard (2004) demonstrated that production errors in aphasics were correlated with the number of phonemes of the word to be pronounced. This effect of phonological length has also been reported in healthy participants in speech production tasks (Levelt, 1992; Sternberg et al., 1980; Meyer et al., 2003; Roelofs, 2002; but see Romani et al., 2010; Bachoud-Lévi et al., $1998^{1}$ ). In pSTM tasks, sequences of short words are better recalled than sequences of long words. For instance

\footnotetext{
${ }^{1}$ It is possible, under certain task demands and with the use of pressure on speed or accuracy, to induce the initiation of articulation before the full planning of the phonological utterance to be pronounced has been completed.
}

a list containing bed, sky, lamp, is better recalled than a list containing crocodile, telephone, magazine. This word-length effect depends on the phonological length number of phonemes, syllables) of the words to be memorized (Caplan et al., 1992; Caplan and Waters, 1994; Service, 1998; Neath and Nairne, 1995) and has been attributed to the phonological output buffer (Jacquemot and Scott, 2006). The length effect is also observed when spoken output is not required, suggesting that it does not result only from delay during output (Baddeley et al., 2002; Cowan et al., 2003; Dosher and $\mathrm{Ma}, 1998)$. In addition, under articulatory suppression, which prevents participants from covertly rehearsing the phonological trace and therefore from using the phonological output buffer, the word-length effect is abolished (Baddeley, 1986).

However the localization of the word-length effect in the subvocal rehearsal component is controversial (Nairne, 1990; Neath and Nairne, 1995; Romani et al., 2005; Nairne et al., 1997; Neath, 2000). According to an alternative view, the standard length effect derives from the number of phonological features to be stored and because of capacity limits, trace decay or interference, longer words are at a disadvantage in recall tasks because more units have to be retained. But, in addition, there is an influence of lexico-semantic factors (Brown and Hulme, 1995; Hulme et al., 1997; Romani et al., 2005). Longer words have an advantage since they offer more residual phonological information from which to attempt reconstruction (or redintegration) and fewer lexical competitors. If just one phonological segment of a word is lost, it would be easy to reconstruct "television" from "tele_sion" but if one segment of a shorter item is lost such as in "_at", it will be difficult to retrieve "cat" between a great number of alternatives (bat, rat, hat, fat, etc.). In these models, the wordlength effect results from the coupled effect of trace decay and of lexico-semantic influence and there is no need of subvocal rehearsal to account for it (Neath and Nairne, 1995; Brown and 
Hulme, 1995; Hulme et al., 1997). In some cases, the effect of lexico-semantic factors becomes more influent than the effect of the number of phonological features, leading to the abolition of the word-length effect. For instance, under articulatory suppression, the self-produced speech adds noise to the phonological buffer. This addition of noise promotes the activation of lexico-semantic representation such as it occurs in irrelevant speech situations (Neath et al., 1998; Nairne, 1990; Gupta and MacWhinney, 1995; Romani et al., 2005) and encourages reliance on lexico-semantic information rather than phonological. As a result, longer words can more easily be reconstructed from degraded phonology than shorter words overriding the effect of the number of phonological features. Therefore, the abolition of length effect results not from the disruption of the rehearsal process but as a consequence of the interference of self-produced speech. Subvocal rehearsal in pSTM becomes an optional part of the system (Romani et al., 2005) or even a non-existing component (Brown and Hulme, 1995; Neath et al., 2003a; Neath and Nairne, 1995).

In this paper, we intend to clarify the issue of the link between the length effect and the potential subvocal rehearsal component. The aim is to decide whether the abolition of the word-length effect under articulatory suppression is only a consequence of self-produced speech or whether it reflects the disruption of subvocal rehearsal. In order to disentangle these two alternatives, we tested two patients who each had a deficit in PSTM. FA presents a conduction aphasia restricted to pseudowords. In FA, the link between phonological input and output is damaged (see Fig. 1), implying a damaged subvocal rehearsal component. This creates a naturally occurring case of articulatory suppression: i.e., the rehearsal process is blocked, but without the interference of self-produced speech. Models in which the word-length effect emerges due to subvocal rehearsal predict that a word-length effect should be absent in FA, since, as in normal subjects under articulatory suppression, there is no possible rehearsal (Jacquemot and Scott, 2006; Baddeley, 2003). Alternative models, however, predict that the word-length effect should be observed in FA, since according to these models, the length effect is independent from articulatory suppression (Romani et al., 2005; Neath et al., 1998). The second patient, FL, acts as a patient control. Indeed, this patient has a reduced short-term memory span, just as FA, but is not impaired in pseudoword repetition. Since FL has a normal rehearsal component, both types of models predict that FL should have a normal length effect.

\section{Case report: FA}

FA is a 54-year-old, right-handed retired secretary (education: 9 years). One year before, she had a stroke leading to conduction aphasia. FA's deficit was extensively studied and described in a previous study (Jacquemot et al., 2007). In the present paper, we report new data relevant to the relationship between word-length effect and subvocal rehearsal.

\subsection{Word comprehension and production}

Word comprehension was tested with a picture matching task requiring to decide whether an auditory word matches a picture. The auditory word could either be the correct one such as the word hat for the picture $e_{\curlywedge}$ of a hat $(N=64)$, a phonological distractor such as rat $(N=64)$, a semantic distractor such as bonnet $(\mathrm{N}=64)$ or a unrelated distractor such as cup $(N=64)$. FA performed very well on this task $(98.5 \%$ correct). FA's word production performance was assessed with a naming task of seventy pictures composed of 20 monosyllabic, 20 bisyllabic and 20 trisyllabic words. For each of these lengths, half of the words were low frequency and half were high frequency. There were also 10 quadrisyllabic words of low frequency. FA's word production was impaired relative to the performance of matched controls $(N=5$, age range 52-60, years of education range 2-12) [respectively $84.3 \%$ and $99.7 \% \pm .6$ correct responses; significance test (Crawford and Howell, 1998) $t=23.4, p$ (two-tailed) $<.001]$. Her errors comprised non-responses $(N=4)$, phonological paraphasia $(N=1)$ and semantic paraphasia $(N=6)$. She also suffered from a minor anarthric deficit characterized by the production of some distorted phonemes and a slow rate of speech. Her digit span was within normal range (4 forward and 5 backward).

\subsection{Conversion mechanisms between phonological input and phonological output}

The conversion mechanism of phonological input into phonological output was assessed with a repetition task of words $(N=32)$ and pseudowords $(N=16)$. There were half monosyllable items and half bisyllable items for both words and pseudowords. For each length (monosyllables and bisyllables) words were half high frequency and half low frequency; likewise, for each length pseudowords were half of low neighbourhood density (no phonological neighbour) or high neighbourhood density (more than 1). FA's performance is reported in Table 1 . She was significantly more impaired in the pseudoword repetition condition than in the word condition [respectively $43.7 \%$ correct and $87.5 \%$ correct; $\chi^{2}(1)=10$,

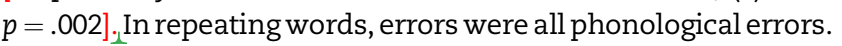
In repeating pseudowords, errors include phonological errors (55\%), lexicalization errors (22\%), non-responses (17\%), and unrelated responses (6\%). There was neither a length effect with words, nor a neighbourhood effect with pseudowords. This discrepancy between performance for words and pseudowords was not observed in controls (respectively, 99.4\% correct and $98.7 \%$ correct) and significantly differs from FA's pattern of results [Revised Standardized Difference Test ${ }_{\Lambda}$ (Crawford and Garthwaite, 2005) $\mathrm{t}(4)=7.6, p$ (two-tailed) $=.002]$ (Fig. 2A).

FA's deficit was not explained by any perceptual deficit: she flawlessly completed a discrimination task that involves phonologically minimal pairs of words and pseudowords (see Table 1). These pairs were recorded by two speakers (male and female) and were used to construct 120 AX trials, half of them being composed by the repetition of the same item coupure/ coupure/kupyr/ and half of them being composed by two items that differ minimally such as coupure/couture/kupyr/ /kutyr/. FA's task was to decide whether the two stimuli were the same word (or pseudoword) or not. She performed at the same level than controls $[96 \%$ correct and $96.5 \%$ correct respectively, significance test (Crawford and Howell, 1998), $t=.1, \quad p \quad$ (two-tailed) $=.92]$. FA's deficit for repeating 
Table 1 - Percent of correct responses for FA, FL and 5 matched controls (age range 51-56, years of education range 2-17).

\begin{tabular}{|c|c|c|c|c|}
\hline & $\mathrm{N}$ of items & FA (\%) & FL (\%) & Controls, $N=5( \pm S D)$ \\
\hline \multicolumn{5}{|c|}{ From perception to production: Repetition } \\
\hline Word & 64 & 87.5 & 98.4 & $99.4 \% \pm .5$ \\
\hline Pseudoword & 32 & 43.7 & 94 & $98.7 \% \pm .9$ \\
\hline \multicolumn{5}{|c|}{ Speech perception: Minimal pair discrimination } \\
\hline Words & 60 & 96.7 & 98.3 & $98.5 \% \pm 6.3$ \\
\hline Pseudoword & 60 & 95 & 96.6 & $94.5 \% \pm 3.3$ \\
\hline \multicolumn{5}{|c|}{ Speech production: Reading } \\
\hline Word & 64 & 91.7 & & $99.3 \% \pm .7$ \\
\hline Pseudoword & 32 & 90.6 & & $97.5 \% \pm 2.6$ \\
\hline \multicolumn{5}{|c|}{ From production to perception: Rhyme judgment on written words } \\
\hline Covert condition & 40 & 82.5 & 97.5 & $96.5 \% \pm 2.9$ \\
\hline Overt condition & 40 & 82.5 & & \\
\hline
\end{tabular}

pseudowords cannot be explained by a deficit in producing pseudoword: FA's reading performance was assessed and even if she was not flawless in reading task (FA: $91.6 \%$ correct, controls: $99.1 \%$ correct, $t=9.8, p=.001$ ), her performance in reading pseudowords was largely better than in repeating pseudowords [respectively, 90.6\% correct and 43.7\% correct; $\left.\chi^{2}(1)=13.9, p<.01\right]$. Reading errors were all phonemic errors and there was neither a length nor a neighbourhood effect. Therefore FA's dramatic difficulties in repeating pseudowords could not be attributed to a deficit of the speech perception system or to a deficit of the speech production system. Given that this deficit specifically affected pseudowords - that do not have any lexico-semantic entry ${ }_{\Lambda}$ - we proposed that FA suffered from an impairment of the phonological mechanism that connects speech perception and the speech production system, that is the mechanism that converts the phonological input code into the phonological output code (see Fig. 1) (Jacquemot et al., 2007).

We further assessed whether the mechanism that converts phonological output into phonological input was spared or not. We used a silent rhyme judgment task in which the patient had to decide if two written words would rhyme if pronounced. FA was asked to perform the task silently. This task, in which strings of letters have to be phonologically compared, requires several steps to be achieved. First, strings of letters have to be converted in a phonological form and stored into the phonological output buffer (Howard and Nickels, 2005; Nickels et al., 1997). Then the phonological form has to be converted into a phonological input for evaluating the rhyme (Burani et al., 1991; Howard and Franklin, 1990). Therefore, accurate performance on this task should reflect the integrity of the phonological output buffer and the conversion mechanism of the phonological output into the phonological input.

We constructed two sets of 20 pairs of written words, rhyming or non-rhyming. Within the 20 rhyming pairs, there were 10 pairs that were also orthographically similar [e.g., mémoire-armoire, (memwaRt- $(\mathrm{aRmwaR})$ memory-wardrobe]

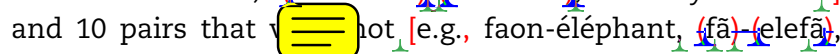
fawn-elephant]; similarty, within the 20 non-rhyming pairs, there were 10 dissimilar pairs [e.g., escalier-râteau, $f_{\Lambda}$ skaljet(Rato) ${ }_{2}$ stairs-rake] and 10 orthographically similar pairs [e.g., fille-tranquille, (fijij-t(tRãkill, girl-peaceful]. Thus, the reliance on orthographic information during this task would lead to errors. FA's performance was lower than control subjects in this task (82.5\% correct and $96.5 \%$ correct respectively, $t=4.4$, $p=.012$ ). As this task requires to covertly read the words, any reading deficit would impact the performance of the rhyme judgment task. We already showed that FA was slightly impaired in reading, thus we further assessed her reading performance on the same words as those used in the rhyming task. The results show that the level of performance on the overt reading task was similar to that on the covert rhyme judgment task (82.5\% correct and $82.5 \%$ correct respectively) suggesting that the errors in the rhyme judgment task resulted from reading difficulties. FA flawlessly performed the rhyming task on the items that she has no difficulty in reading.

This pattern of performance suggests that the phonological output buffer and the conversion mechanism from phonological output into phonological input are spared in FA whereas the conversion mechanism from phonological input to phonological output is impaired (Jacquemot et al., 2007). In the present study, we tested FA with two additional tasks specifically tapping into the two components of PSTM: the phonological input buffer with a task involving to memorize lists of phonological similar and dissimilar items and subvocal rehearsal with a task involving to memorize lists of short and long words.

\subsection{Phonological similarity effect}

The phonological similarity effect is not affected by the disruption of subvocal rehearsal component (Vallar and Baddeley, 1984b) meaning that FA should demonstrate the typical phonological similarity effect.

\subsubsection{Methods}

Immediate serial recall of phonologically similar and dissimilar items was assessed. FA was instructed to listen to a list of items and to recall them in the correct order. FA was taught to associate two auditory $\mathrm{CV}-\mathrm{CV}$ pseudowords (C, consonant; $\mathrm{V}$, vowel) to keys "1" and "2", respectively, on a computer keyboard. During a prior training session, FA was asked to choose the correct key according to each of the pseudowords presented in isolation. After training, FA was presented with 

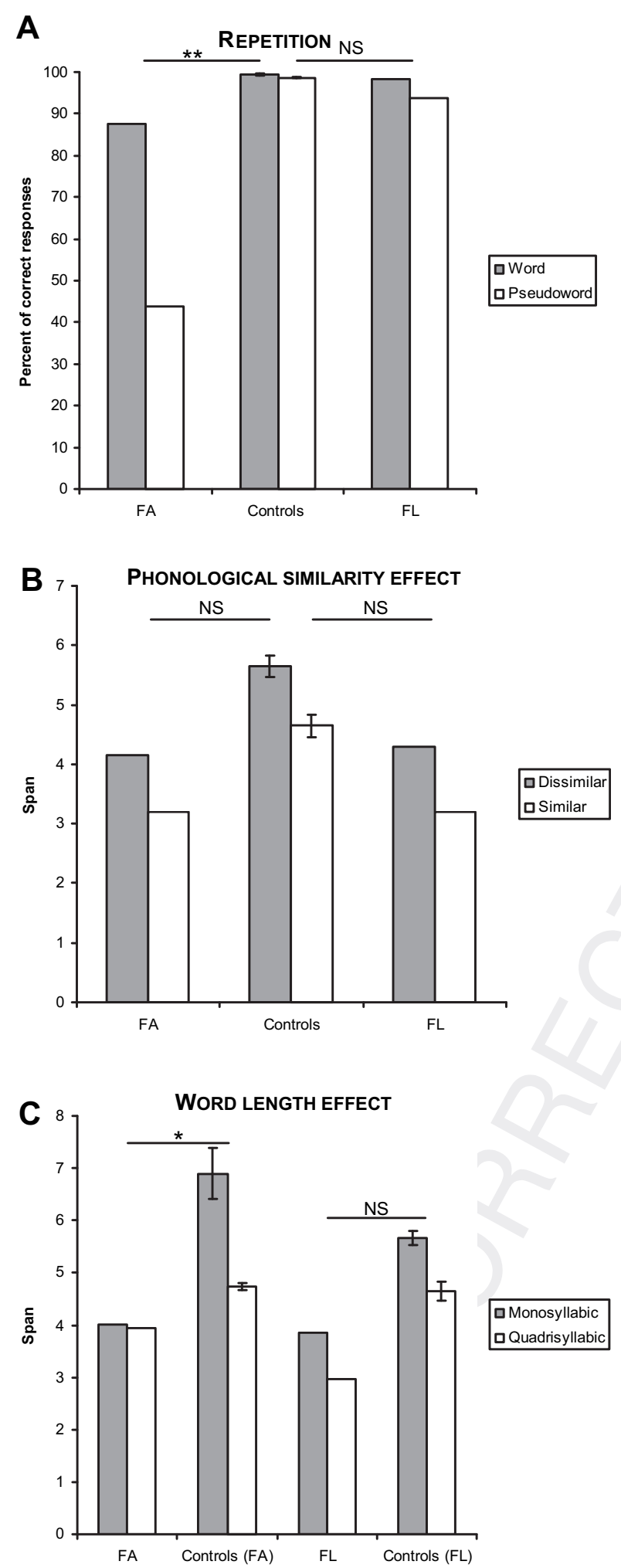

Fig. 2 - Performance (mean and standard error) of FA, FL and control participants. NS for non-significant, ${ }^{*} p<.05$, ${ }^{* *} p<.01$. A) Percent of correct responses in the repetition task. B) Estimated span for dissimilar and similar items. C) Estimated span for monosyllabic and quadrisyllabic words. Because the task procedure was slightly different for testing the word-length effect in FA and in FL, two groups of controls were tested according to each procedure. progressively longer, random sequences of the two pseudowords. FA was required to recall the sequence and transcribe it with the keys " 1 " and "2". Six trials of two, three, four, five, and six items were presented in ascending order (for procedure details see Jacquemot et al., 2006). For each pseudoword, six acoustically different tokens were used in order to constrain the participants to use the phonological information and not the acoustic one. The experiment was divided into two parts. In the first part, the two pseudowords were phonologically dissimilar-/rapi/ versus /foga/-while in the second part they were phonologically similar-/mipa/versus /miba/-and constituted a minimal pair differing in only one distinctive phonetic feature (i.e., voicing).

\subsubsection{Results}

Results were analysed by estimating the critical sequence length that yielded $50 \%$ of correct recall for each condition (dissimilar and similar items). We call this sequence length the estimated memory span. ${ }^{2}$ FA's data was compared to data of 10 matched control participants (age range 50-55, years of education range $5-22$ ). FA's span is 4.15 for the dissimilar items and 3.2 for the similar items. The estimated span of the control participants is 5.7 for the dissimilar items and 4.6 for the similar items (Fig. 2B). Controls show the typical phonological similarity effect $[\mathrm{t}(9)=$ 9.9, $p<.001]$. The comparison of FA and controls' results was computed with RSDT (Crawford and Garthwaite, 2005). FA's Q span is lower than controls' span for both dissimilar condition $[\mathrm{t}(9)=2.5, p$ (two-tailed) $=.046]$ and similar condition $[\mathrm{t}(9)=2.3$, $p($ two-tailed $)=.03]$ but the performance difference between the similar and dissimilar items is not different to controls $[t(9)=.37$, $p$ (two-tailed $)=.72]$, meaning that FA as controls shows the typical phonological similarity effect.

\subsection{Word-length effect}

\subsubsection{Methods}

Immediate serial recall of short and long words was assessed. FA was instructed to listen to a list of words and to recall them in the correct order. Words were presented at the rate of $1 / \mathrm{sec}$. Two sets of stimuli, one composed of 10 monosyllabic words, and another of 10 quadrisyllabic words, were used. Monosyllabic words were phonologically less complex in terms of number of syllables and phonemes than quadrisyllabic words. The words in the two sets were concrete words of high imaginability and were matched for frequency.

For each set (monosyllabic and quadrisyllabic words), ten trials of a given sequence length (sequences from two to ten words) were generated at random. They were presented in ascending order. No word was presented twice within a sequence. If FA's recall of the first three trials of a given sequence length was correct, we assumed that this length was within her span and proceeded to the following string length (Vallar and Baddeley, 1984a). FA was required to immediately recall the words presented by the examiner by pointing to the appropriate stimuli in the presentation order. The ten words were presented in written format only during the recall phase.

\footnotetext{
${ }^{2}$ This estimated span was computed by regressing the average recall rate across sequence length with a linear function. This was done in $\mathrm{R}$ by fitting the data of each individual participant.
} 
This procedure was used because, due to FA's mild anarthria, her speech production was sometimes slowed and that may have affected her STM's performance (Papagno et al., 2008).

\subsubsection{Results}

Results were analysed as above by estimating the span, that is the critical sequence length that yielded $50 \%$ of correct recall for each condition (monosyllabic and quadrisyllabic words). FA's data was compared to the data of 5 matched control participants (age range 52-60, years of education range 2-12) tested with the same modality of response (pointing). FA's span is 4 for the monosyllabic words and 3.9 for the quadrisyllabic words (Fig. 2C). Controls' span is 6.9 for the monosyllabic words and 4.7 for the quadrisyllabic words. The controls show the typical word-length effect $[t(4)=5.1, p=.007]_{\Omega}$ The comparison of FA and controls' results using RSDT shows that FA's span for monosyllabic words is marginally lower than controls' span $[\mathrm{t}(4)=2.4, p$ (two-tailed) $=.06]$ and that FA's span for quadrisyllabic words is lower than controls $[t(4)=4.8, p$ (twotailed) $=.008]$. The crucial point is that FA's difference between monosyllabic and quadrisyllabic words is significantly different from that observed in controls $[t(4)=4.2, p \text { (two-tailed) }=.014]_{,}$ showing that contrary to controls, FA does not display the typical word-length effect.

\subsection{Discussion}

Two tasks were used for assessing the phonological input buffer and the subvocal rehearsal component. First, like controls, FA shows a phonological similarity effect, that is better performance in memorizing sequences of dissimilar items compared to sequences of similar items. FA's span is lower than controls, suggesting a deficit of the phonological input buffer. Secondly, tested with sequences of short and long words, FA, contrary to controls, does not show any word-length effect.

We assessed the phonological similarity effect and the length effect with auditory stimuli and did not investigate these two effects in the visual modality. This restriction is explained by the fact that FA's performance in reading was not perfect, suggesting a mild deficit of the orthography-tophonology conversion process. An abnormal phonological similarity word-length effect with visual stimuli would have been difficult to interpret, given that part of the effect could be the result of the impairment in orthography-to-phonology conversion and not related to the phonological buffer or phonological rehearsal per se. Hence, we only tested FA with auditory stimuli, which do not require mediation with orthography, and directly measure pSTM.

In order to ensure that the absence of length effect results from the subvocal rehearsal disruption and not from the phonological input buffer deficit, we tested another patient, FL, who suffers from a deficit of the phonological input buffer, but does not have any impairment of the conversion mechanism of phonological input into phonological output.

\section{Case report: FL}

Patient FL is a right-handed 59-year-old computer specialist. Five years before participating in the study, he had a stroke leading to global aphasia. FL's deficit was previously described in Jacquemot et al. (2006). A computed tomography (CT) scan (at admission) and a magnetic resonance imaging (MRI) scan (4 years later) confirmed a left perisylvian stroke. He was fluent in speech production, to the extent that uninformed listeners did not detect abnormalities in their speech. He performs well in the naming task $(95 \%$ with only semantic errors such for instance skirt for dress). His word comprehension was evaluated with the same picture matching task as for FA and FL flawlessly performed this task (100\% correct).

\subsection{Conversion mechanism between phonological input and phonological output}

As FA, FL was tested with a phonological discrimination task that involves minimal pairs of words and pseudowords. FL flawlessly performed the discrimination task showing that he had no phonological decoding deficit. We also assessed the conversion mechanism from phonological input into phonological output with the same repetition task used with FA. FL's performance does not differ from controls $s_{\lambda}[97 \%$ correct and $99 \%$ correct respectively, $t=.84, p$ (two-tailed) $=.4]$. FL performs equally with words and pseudowords $\left[\chi^{2}(1)=1.6\right.$, $p=.2]$ (Fig. 2A). We assessed the conversion mechanism from phonological output to phonological input with the silent rhyme judgment task used with FA. FL performs as well as controls $(97.5 \%$ correct and $96.5 \%$ correct respectively, $t=.3$, $p=.7$ ) (see Table 1).

This overall pattern of performance suggests that the both conversion mechanisms between phonological input and output and the phonological output buffer are intact in FL.

\subsection{Phonological similarity effect}

In order to assess the phonological similarity effect in $\mathrm{FL}$, we used the same experimental task as used with FA and results were analysed according to the same method. The estimated FL's span is 4.3 for dissimilar items and 3.2 for similar items (Fig. 2B). FL's data was compared to the data of 10 matched control participants (age range 50-55, years of education range 5-22). The estimated span of the control participants is 5.7 for the dissimilar items and 4.65 for the similar items. Controls show the typical phonological similarity effect $[\mathrm{t}(9)=$ 9.94, $p<.001]_{\Lambda}$ RSDT analysis shows that FL's span is lower than controls' span for both dissimilar condition $[t(9)=2.3$, $p$ (two-tailed) $=.04]_{\Lambda}$ and similar condition $[\mathrm{t}(9)=2.2, p$ (twotailed $=.04]$ but that the span difference between similar and dissimilar items is the same as in controls $[\mathrm{t}(9)=.17, p$ (twotailed) $=.86]$. This data shows that FL, like controls, shows the typical phonological similarity effect but that his phonological input buffer capacity is reduced relative to controls.

\subsection{Word-length effect}

Immediate serial recall of short and long words was assessed. FL was instructed to listen to a list of words and to recall them in the correct order. The material and the testing procedure were similar to that used with FA except that in the recall phase, FL was asked to orally repeat the sequence instead of pointing to the written stimuli in the correct order. 
We estimated FL's span (the critical sequence length that yielded $50 \%$ of correct recall) for the monosyllabic and quadrisyllabic conditions. FL's results were compared to the data of 10 matched control participants (age range 50-55, years of education range 5-22) tested with the same modality of recall (oral response). FL's span is 3.86 for the monosyllabic words and 2.97 for the quadrisyllabic words (Fig. 2C). The estimated span of the control participants is 5.7 for the monosyllabic words and 4.6 for the quadrisyllabic words. The controls show the typical word-length effect $[t(9)=6.82, p<.001]$. FL's span is lower than controls' span, for both monosyllabic words $[\mathrm{t}(9)=$ 3.8, $p$ (two-tailed $)=.004]_{\Lambda}$ and quadrisyllabic words $[t(9)=2.9$, $p$ (two-tailed $)=.016]$, but the difference between the span of the monosyllabic and quadrisyllabic words is similar to that obtained in controls $\mathrm{s}_{\wedge}[t(9)=.8, p$ (two-tailed) $=.4]$ indicating that FL shows the typical word-length effect.

\subsection{Discussion}

FL's results show that word comprehension and word production systems are both unimpaired, and that the two conversion mechanisms between phonological input and phonological output are both intact. In the memory tasks, FL's span is globally lower than controls' span: his performance in the phonological similarity task is lower than the controls and his performance in the word-length task is lower than controls showing that FL's phonological input buffer has a lower storage capacity. Nevertheless, like controls, FL presents the typical span difference between similar and dissimilar items and the typical span difference between the monosyllabic and quadrisyllabic items. Overall FL suffers from an input buffer deficit with no associated deficit of the conversion mechanisms between phonological input and output.

Both FA and FL show a phonological input buffer deficit with a lower storage capacity relative to controls. The difference between FA and FL is that FA also suffers from a deficit of the mechanism that converts the phonological input into the phonological output. FL shows a word-length effect whereas FA does not. FL was tested in order to assess whether the absence of word-length effect could be explained by the phonological input buffer deficit. Indeed, a reduction of the phonological input buffer capacities could trigger the use of the lexico-semantic information for memorizing the words. With low phonological memory abilities the system could rely on the lexico-semantic level rather than the phonological to store the information. According to such hypothesis, the length effect could disappear not because of the disruption of subvocal rehearsal but because of the activation of the lexicosemantic memory which is not sensitive to length effect. FL's result does not confirm this hypothesis and suggests that the absence of word-length effect could not solely be explained by a phonological input buffer deficit.

This is in accordance with previous data on two patients GF and CM with "locked in syndrome" (Vallar and Cappa, 1987; Cubelli and Nichelli, 1992). These patients were unable to produce any speech output and to covertly rehearse speech sounds (see Cubelli et al., 1993). GF and CM did not suffer from any comprehension deficit, their digit span was within normal limits and GF showed normal performance in probe recognition task using auditory material suggesting that his phonological input buffer was not impaired. Interestingly these patients that could not use their subvocal rehearsal showed a phonological similarity effect but no word-length effect. Their results showed that the absence of length effect in memory task was observed in natural situation of the abolition of subvocal rehearsal without interference of selfproduced speech.

\section{General discussion}

We tested two patients, FA and FL. FA suffered from a conduction aphasia with a reduction of short-term memory and a relatively specific impairment in pseudoword repetition, with almost preserved comprehension, production, and word repetition. FL, in contrast was only impaired in short-term memory. FA's deficit results from selective damage of the link between the input and output phonological codes, an element of the subvocal rehearsal component (Baddeley, 2003; Jacquemot and Scott, 2006). Tested on a sequence recall task, FA shows a normal phonological similarity effect: as controls and FL, she better recalls items that are phonologically dissimilar compared to phonologically similar ones. In contrast, FA does not show any word-length effect: her span does not differ for monosyllabic and quadrisyllabic words. This result differs from controls and from FL: they show a typical word-length effect meaning that the absence of word-length effect in FA could not be due to experimental testing conditions. FL's data confirmed that the absence of the word-length effect in FA could not be resulting solely from the phonological input buffer deficit.

Two main types of pSTM models are proposed to explain the phonological similarity and word-length effects. There are clear differences between these two types of models with respect to the role of subvocal rehearsal in pSTM. In the first type, subvocal rehearsal is involved in refreshing the phonological trace that is stored in the phonological buffer through its access to the phonological output buffer (Baddeley, 2003; Howard and Franklin, 1990; Howard and Nickels, 2005; Nickels et al., 1997; Monsell, 1987; Murphy et al., 2006) and its disruption induces the abolition of the word-length effect. In the second type of pSTM model, subvocal rehearsal is only an optional component of the system. The length effect results from the combined effect of phonological and lexicosemantic factors and the depletion of word-length effect under articulatory suppression is a consequence of the interference of self-produced speech (Neath et al., 1998, 2003b; Gupta and MacWhinney, 1995; Campoy and Baddeley, 2008; Romani et al., 2005).

FA presents a natural case of articulatory suppression without any disturbance of additional factors such as selfproduced speech or dual task interference. According to model with optional rehearsal, she should exhibit a wordlength effect. But this is not the case: FA's results fit with pSTM model in which subvocal rehearsal is one of the main components of pSTM (Howard and Franklin, 1990; Nickels et al., 1997; Baddeley, 2003; Jacquemot and Scott, 2006). In this type of models, pSTM performance depends on the capacities of both phonological input and phonological output 
buffers and on the ability to convert phonological information between them (Fig. 1). Because of her deficit, FA can only use the phonological input buffer to perform $a_{\Lambda}$ short-term memory task. As a result, she only shows a phonological similarity effect, not a length effect. In other words, FA's results are close to those of normal controls under an articulatory suppression task. This suggests that under articulatory suppression, even if the self-produced speech may impact pSTM performance (Gupta and MacWhinney, 1995), it cannot be entirely responsible for the depletion of the length effect which mainly results from the disruption of subvocal rehearsal.

Our data supports the hypothesis that the length effect emerges at least partly from the phonological output buffer, a finding also supported by sign language literature which reports a length effect in memory tasks when using short and long signs (Wilson and Emmorey, 1998). This is in favor of models of pSTM that include two phonological buffers, the input and output buffers. Articulatory suppression has at least two separate effects on the components of pSTM: (1) it disrupts the rehearsal component and (2) it interferes with the phonological input buffer (Gupta and MacWhinney, 1995). This could explain why the word-length effect is even found to be inverted under articulatory suppression (Romani et al., 2005). Because of the interference of self-produced speech on the phonological input process, articulatory suppression promotes the activation of the lexico-semantic level that is not sensitive to length. At this level, longer words have an advantage since they offer more residual information from which reconstruction can be attempted (Hulme et al., 1997), explaining why an inversion of length effect could be observed.

Previous findings also reported that the length effect could survive under articulatory suppression when the memory task involves pseudowords and not words (Romani et al., 2005). We propose that this pseudoword length effect under articulatory suppression may result from the interference of the self-produced speech. This auditory signal impacts the process of phonological decoding and is likely to degrade the stored phonological input. Because of this phonological interference, some phonemes may be decoded misguidedly, and the more phonemes that compose the pseudowords, the more chance there is of making phonemic errors. Consequently, long words have a greater chance of giving rise to phonological errors and thus errors in memory tasks. This could explain why a pseudoword length effect could survive under articulatory suppression. FA's deficit allows for the testing of the impact of subvocal rehearsal disruption on length effect without the interference of self-produced speech. FA's data shows that when subvocal rehearsal is specifically disrupted the length effect disappears.

Before closing, we propose a speculative account of why the length effect should be related to the phonological output buffer rather than to the input buffer. According to Cowan (2001), the phonological input buffer stores a given number of units, called chunks, that can either be phonemes, syllables, words or groups of words (Cowan, 2001). The number of chunks that can be memorized is limited to 3 or 5 (Chen and Cowan, 2009). In this framework, the only way to increase pSTM storage capacity is to extend the size of chunks by grouping syllables or words into larger chunks (Chen and Cowan, 2005). In our experiment, we presented the spoken stimuli as isolated words, each of them being uttered as a prosodic unit. We hypothesize that subvocal rehearsal enables participants to re-chunk the list of words by building larger multiword prosodic units, thus increasing the size of chunks. In essence, this is exactly the same as in speech production where the output buffer is devoted to stringing together phonemes to form a hierarchy of units, syllables, feet, words, phonological phrases (Levelt, 1992; Levelt et al., 1999; Jacquemot and Scott, 2006). Hypothetically, in a pSTM experiment, the same process would be involved for binding together syllables or words to form a new chunk, increasing memory capacity. But a chunk has a limited size (Cowan, 2010) and if the units are too large they cannot be combined into a chunk. According to this account, the shorter the words are, the more of them can be bound into a chunk, which explains why performance differs for sequences of short and long words (Glanzer and Razel, 1974). Thus the phonological output buffer may allow for increasing the length of the chunks and would be at least partly responsible for the length effect. This might explain why the phonological input buffer would be more sensitive to the number of chunks whereas the output buffer would be more sensitive to the size of chunks.

In conclusion, these data suggest that the phonological output buffer plays a role in pSTM and is at least partly responsible for the word-length effect. This predicts that patients with a specific phonological output buffer deficit should present the same pattern as those suffering from a damage of the link between the input and output buffers, that is a normal phonological similarity effect but no length effect. Further studies comparing patients with phonological input buffer deficits to patients with phonological output deficits would provide interesting data to disentangle the role of each buffer in the phonological similarity and phonological length effects and their relation with the number and the size of chunks.

\section{Acknowledgments}

The authors are grateful to John Crawford for providing the statistical tools for analysing single cases in neuropsychology. The authors are also grateful to Roberto Cubelli and two anonymous reviewers for their many helpful comments.

\section{R E F E R E N C E S}

Bachoud-Lévi A-C, Cohen L, Dupoux E, and Mehler J. Where is the length effect? A cross-linguistic study of speech production. Journal of Memory and Language, 39: 331-346, 1998.

Baddeley A. Working Memory. New York: Oxford University Press, 1986.

Baddeley A. Working memory: Looking back and looking forward. Nature Review Neuroscience, 4: 829-839, 2003.

Baddeley A, Chincotta D, Stafford L, and Turk D. Is the word length effect in STM entirely attributable to output delay? Evidence from serial recognition. Quarterly Journal of Experimental Psychology A, 55: 353-369, 2002. 
Baddeley A and Hitch G. Working memory. In Bower GA (Ed), The Psychology of Learning and Motivation. New York: Academic Press, 1974.

Baddeley A, Lewis V, and Vallar G. Exploring the articulatory loop. Quarterly Journal of Experimental Psychology A, 36: 233-252, 1984.

Brown GDA and Hulme C. Modeling item length effects in memory span: No rehearsal needed? Journal of Memory and Language, 34: 594-621, 1995.

Burani C, Vallar G, and Bottini G. Articulatory coding and phonological judgements on written words and pictures: The role of the phonological output buffer. The European Journal of Cognitive Psychology, 3: 379-398, 1991.

Campoy G and Baddeley A. Phonological and semantic strategies in immediate serial recall. Memory, 16: 329-340, 2008.

Caplan D, Rochon E, and Waters GS. Articulatory and phonological determinants of word length effects in span tasks. Quarterly Journal of Experimental Psychology A, 45: 177-192, 1992.

Caplan D and Waters GS. Articulatory length and phonological similarity in span tasks: A reply to Baddeley and Andrade. Quarterly Journal of Experimental Psychology A, 47: 1055-1062, 1994.

Caramazza A, Miceli G, and Villa G. The role of the (output) phonological buffer in reading, writing, and repetition. Cognitive Neuropsychology, 3: 37-76, 1986.

Chen $\mathrm{Z}$ and Cowan N. Chunk limits and length limits in immediate recall: A reconciliation. Journal of Experimental Psychology: Learning, Memory, and Cognition, 31: 1235-1249, 2005.

Chen $\mathrm{Z}$ and Cowan N. Core verbal working-memory capacity: The limit in words retained without covert articulation. Quarterly Journal of Experimental Psychology (Colchester), 62: 1420-1429, 2009.

Cowan N. The magical number 4 in short-term memory: A reconsideration of mental storage capacity. Behavioral and Brain Sciences, 24: 87-114 [discussion 114-185], 2001.

Cowan N. The magical mystery four: How is working memory capacity limited, and why? Current Directions in Psychological Science, 19: 51-57, 2010.

Cowan N, Baddeley AD, Elliott EM, and Norris J. List composition and the word length effect in immediate recall: A comparison of localist and globalist assumptions. Psychonomic Bulletin \& Review, 10: 74-79, 2003.

Crawford JR and Garthwaite PH. Testing for suspected impairments and dissociations in single-case studies in neuropsychology: Evaluation of alternatives using Monte Carlo simulations and revised tests for dissociations. Neuropsychology, 19: 318-331, 2005.

Crawford JR and Howell DC. Comparing an individual's test score against norms derived from small samples. The Clinical Neuropsychologist, 12: 482-486, 1998.

Cubelli R and Nichelli P. Inner speech in anarthria: Neuropsychological evidence of differential effects of cerebral lesions on subvocal articulation. Journal of Clinical and Experimental Neuropsychology, 14: 499-517, 1992.

Cubelli R, Nichelli P, and Pentore R. Anarthria impairs subvocal counting. Perceptual and Motor Skills, 77: 971-978, 1993.

Dosher BA and Ma JJ. Output loss or rehearsal loop? Outputtime versus pronunciation-time limits in immediate recall for forgetting-matched materials. Journal of Experimental Psychology: Learning, Memory and Cognition, 24: 316-335, 1998.

Glanzer M and Razel M. The size of the unit in short-term storage. Journal of Verbal Learning and Verbal Behavior, 13: 114-131, 1974.

Gupta P and MacWhinney B. Is the articulatory loop articulatory or auditory? Re-examining the effects of concurrent articulation on immediate serial recall. Journal of Memory and Language, 34: 63-88, 1995.
Howard D and Franklin S. Memory without rehearsal. In Vallar G and Shallice $\mathrm{T}$ (Eds), Neuropsychological Impairments of Shortterm Memory. Cambridge: Cambridge University Press, 1990.

Howard D and Nickels L. Separating input and output phonology: Semantic, phonological, and orthographic effects in shortterm memory impairment. Cognitive Neuropsychology, 22: 42-77, 2005.

Hulme C, Roodenrys S, Schweickert R, Brown GD, Martin M, and Stuart G. Word-frequency effects on short-term memory tasks: Evidence for a redintegration process in immediate serial recall. Journal of Experimental Psychology: Learning, Memory and Cognition, 23: 1217-1232, 1997.

Jacquemot C, Dupoux E, and Bachoud-Lévi AC. Breaking the mirror: Asymmetrical disconnection between the phonological input and output codes. Cognitive Neuropsychology, 24: 3-22, 2007.

Jacquemot C, Dupoux E, Decouche O, and Bachoud-Lévi AC. Misperception in sentences but not in words: Speech perception and the phonological buffer. Cognitive Neuropsychology, 23: 949-971, 2006.

Jacquemot C and Scott SK. What is the relationship between phonological short-term memory and speech processing? Trends in Cognitive Science, 10: 480-486, 2006.

Laganaro $\mathrm{M}$ and Alario FX. On the locus of syllable frequency effect in speech production. Journal of Memory and Language, 55: 178-196, 2006.

Levelt WJ. Accessing words in speech production: Stages, processes and representations. Cognition, 42: 1-22, 1992.

Levelt WJM, Roelofs APA, and Meyer AS. A theory of lexical access in speech production. Behavioral and Brain Sciences, 22: 1-37, 1999.

Martin R, Lesch M, and Bartha M. Independence of input and output phonology in word processing and short-term memory. Journal of Memory and Language, 41: 3-29, 1999.

Meyer AS, Roelofs A, and Levelt WJ. Word length effects in object naming: The role of a response criterion. Journal of Memory and Language, 48: 131-147, 2003.

Monsell S. On the relation between lexical input and output pathways of speech. In Allport A, Mackay DG, Prinz W, and Scheerer E (Eds), Language Perception and Production: Common Processes in Listening, Speaking, Reading and Writing. London: Academic Press, 1987: 273-311.

Murphy K, Roodenrys S, and Fox A. Event-related potential correlates of the word length effect in working memory. Brain Research, 1112: 179-190, 2006.

Nairne JS. A feature model of immediate memory. Memory and Cognition, 18: 251-269, 1990.

Nairne JS, Neath I, and Serra M. Proactive interference plays a role in the word-length effect. Psychonomic Bulletin \& Review, 4: 541-545, 1997.

Neath I. Modeling the effects of irrelevant speech on memory. Psychonomic Bulletin \& Review, 7: 403-423, 2000.

Neath I, Bireta TJ, and Surprenant AM. The time-based word length effect and stimulus set specificity. Psychonomic Bulletin \& Review, 10: 430-434, 2003a.

Neath I, Farley LA, and Surprenant AM. Directly assessing the relationship between irrelevant speech and articulatory suppression. Quarterly Journal of Experimental Psychology, 56A: 1269-1278, 2003b.

Neath I and Nairne JS. Word-length effects in immediate memory: Overwriting trace decay theory. Psychonomic Bulletin \& Review, 2: 429-441, 1995.

Neath I, Surprenant AM, and LeCompte DC. Irrelevant speech eliminates the word length effect. Memory and Cognition, 26: $343-354,1998$.

Nickels L, Howard D, and Best W. Fractionating the articulatory loop: Dissociations and associations in phonological recoding in aphasia. Brain and Language, 56: 161-182, 1997. 
Papagno C, Lucchelli F, and Vallar G. Phonological recoding, visual short-term store and the effect of unattended speech: Evidence from a case of slowly progressive anarthria. Cortex, 44: 312-324, 2008.

Roelofs A. Syllable structure effects turn out to be word length effects: Comment on santiago et al. (2000). Language and Cognitive Processes, 17: 1-13, 2002.

Romani C, Galluzzi C, and Olson A. Phonological-lexical activation: A lexical component or an output buffer? Evidence from aphasic errors. Cortex, 2010.

Romani C, McAlpine S, Olson A, Tsouknida E, and Martin R. Length, lexicality, and articulatory suppression in immediate recall: Evidence against the articulatory loop. Journal of Memory and Language, 52: 398-415, 2005.

Service E. The effect of word length on immediate serial recall depends on phonological complexity, not articulatory duration. Quarterly Journal of Experimental Psychology, 51: $283-304,1998$.
Sternberg S, Wright CE, Knoll RL, and Monsell S. Motot programs in rapid speech: Additional evidence. In Cole RA (Ed), Perception and Production of Fluent Speech. Hillsdale, NJ: Erlbaum, 1980: 469-505.

Vallar G and Baddeley A. Fractionation of working memory: Neuropsychological evidence for a phonological short-term store. Journal of Verbal Learning and Verbal Behavior, 23: 151-161, 1984a.

Vallar G and Baddeley A. Phonological short-term store, phonological processing and sentence comprehension: A neuropsychological case study. Cognitive Neuropsychology, 1: 121-141, 1984b.

Vallar G and Cappa S. Articulation and verbal short-term memory: Evidence from anarthria. Cognitive Neuropsychology, 4: 55-78, 1987.

Wilson M and Emmorey K. A "Word length effect" for sign language: Further evidence for the role of language in structuring working memory. Memory \& Cognition, 26: 584-590, 1998. 